\title{
A NOTE ON INDUCED RAMSEY NUMBERS
}

\author{
DAVID CONLON, DOMINGOS DELLAMONICA JR., STEVEN LA FLEUR, VOJTĚCH RÖDL, \\ AND MATHIAS SCHACHT \\ Dedicated to the memory of Jirka Matoušek
}

\begin{abstract}
The induced Ramsey number $r_{\text {ind }}(F)$ of a $k$-uniform hypergraph $F$ is the smallest natural number $n$ for which there exists a $k$-uniform hypergraph $G$ on $n$ vertices such that every two-coloring of the edges of $G$ contains an induced monochromatic copy of $F$. We study this function, showing that $r_{\text {ind }}(F)$ is bounded above by a reasonable power of $r(F)$. In particular, our result implies that $r_{\text {ind }}(F) \leqslant 2^{2^{c t}}$ for any 3-uniform hypergraph $F$ with $t$ vertices, mirroring the best known bound for the usual Ramsey number. The proof relies on an application of the hypergraph container method.
\end{abstract}

\section{§1. INTRODUCTION}

The Ramsey number $r(F ; q)$ of a $k$-uniform hypergraph $F$ is the smallest natural number $n$ such that every $q$-coloring of the edges of $K_{n}^{(k)}$, the complete $k$-uniform hypergraph on $n$ vertices, contains a monochromatic copy of $F$. In the particular case when $q=2$, we simply write $r(F)$. The existence of $r(F ; q)$ was established by Ramsey in his foundational paper [17] and there is now a large body of work studying the Ramsey numbers of graphs and hypergraphs. For a recent survey, we refer the interested reader to [5].

In this paper, we will be concerned with a well-known refinement of Ramsey's theorem, the induced Ramsey theorem. We say that a $k$-uniform hypergraph $F$ is an induced subgraph of another $k$-uniform hypergraph $G$ if $V(F) \subset V(G)$ and any $k$ vertices in $F$ form an edge if and only if they also form an edge in $G$. The induced Ramsey number $r_{\text {ind }}(F ; q)$ of a $k$-uniform hypergraph $F$ is then the smallest natural number $n$ for which there exists a $k$-uniform hypergraph $G$ on $n$ vertices such that that every $q$-coloring of the edges of $G$ contains an induced monochromatic copy of $F$. Again, in the particular case when $q=2$, we simply write $r_{\text {ind }}(F)$.

For graphs, the existence of induced Ramsey numbers was established independently by Deuber [6], Erdős, Hajnal, and Pósa [8], and Rödl [18], while for $k$-uniform hypergraphs with $k \geqslant 3$ their existence was shown independently by Nešetřil and Rödl [16] and Abramson

The first author was supported by a Royal Society University Research Fellowship.

The fourth author was partially supported by NSF grants DMS-1102086 and DMS-1301698.

The fifth author was supported through the Heisenberg-Programme of the DFG. 
and Harrington [1]. The bounds that these original proofs gave on $r_{\text {ind }}(F ; q)$ were enormous. However, at that time it was noted by Rödl (unpublished) that for bipartite graphs $F$ the induced Ramsey numbers are exponential in the number of vertices. Moreover, it was conjectured by Erdös [7] that there exists a constant $c$ such that every graph $F$ with $t$ vertices satisfies $r_{\text {ind }}(F) \leqslant 2^{c t}$. If true, the complete graph would show that this is best possible up to the constant $c$. A result of Conlon, Fox, and Sudakov [3], building on earlier work by Kohayakawa, Prömel, and Rödl [13], comes close to establishing this conjecture, showing that

$$
r_{\text {ind }}(F) \leqslant 2^{c t \log t} \text {. }
$$

However, the method used to prove this estimate only works in the 2-color case. For $q \geqslant 3$, the best known bound, due to Fox and Sudakov [11], is $r_{\text {ind }}(F ; q) \leqslant 2^{c t^{3}}$, where $c$ depends only on $q$.

In this note, we study the analogous question for hypergraphs, showing that the induced Ramsey number is never significantly larger than the usual Ramsey number. Our main result is the following.

Theorem 1. Let $F$ be a k-uniform hypergraph with $t$ vertices and $\ell$ edges. Then there are positive constants $c_{1}, c_{2}$, and $c_{3}$ such that

$$
r_{\text {ind }}(F ; q) \leqslant 2^{c_{1} k \ell^{3} \log (q t \ell)} R^{c_{2} k \ell^{2}+c_{3} t \ell},
$$

where $R=r(F ; q)$ is the classical q-color Ramsey number of $F$.

Define the tower function $t_{k}(x)$ by $t_{1}(x)=x$ and, for $i \geqslant 1, t_{i+1}(x)=2^{t_{i}(x)}$. A seminal result of Erdös and Rado [9] says that

$$
r\left(K_{t}^{(k)} ; q\right) \leqslant t_{k}(c t)
$$

where $c$ depends only on $k$ and $q$. This yields the following immediate corollary of Theorem 1.

Corollary 1. For any natural numbers $k \geqslant 3$ and $q \geqslant 2$, there exists a constant $c$ such that if $F$ is a $k$-uniform hypergraph with $t$ vertices, then

$$
r_{\text {ind }}(F ; q) \leqslant t_{k}(c t)
$$

A result of Erdős and Hajnal (see, for example, Chapter 4.7 in [12] and [4]) says that

$$
r\left(K_{t}^{(k)} ; 4\right) \geqslant t_{k}\left(c^{\prime} t\right)
$$

where $c^{\prime}$ depends only on $k$. Therefore, the Erdös-Rado bound is sharp up to the constant $c$ for $q \geqslant 4$. By taking $F=K_{t}^{(k)}$, this also implies that Corollary 1 is tight up to the 
constant $c$ for $q \geqslant 4$. Whether it is also sharp for $q=2$ and 3 depends on whether $r\left(K_{t}^{(k)}\right) \geqslant t_{k}\left(c^{\prime} t\right)$, though determining if this is the case is a famous, and seemingly difficult, open problem.

The proof of Theorem 1 relies on an application of the hypergraph container method of Saxton and Thomason [20] and Balogh, Morris, and Samotij [2]. In Ramsey theory, the use of this method was pioneered by Nenadov and Steger [14] and developed further by Rödl, Ruciński, and Schacht [19] in order to give an exponential-type upper bound for Folkman numbers. Our modest results are simply another manifestation of the power of this beautiful method.

\section{§2. Proof of TheOrem 1}

In order to state the result we first need some definitions. Recall that the degree $d(\sigma)$ of a set of vertices $\sigma$ in a hypergraph $H$ is the number of edges of $H$ containing $\sigma$, while the average degree is the average of $d(v):=d(\{v\})$ over all vertices $v$.

Definition 2. Let $H$ be an $\ell$-uniform hypergraph of order $N$ with average degree $d$. Let $\tau>0$. Given $v \in V(H)$ and $2 \leqslant j \leqslant \ell$, let

$$
d^{(j)}(v)=\max \{d(\sigma): v \in \sigma \subset V(H),|\sigma|=j\} .
$$

If $d>0$, define $\delta_{j}$ by the equation

$$
\delta_{j} \tau^{j-1} N d=\sum_{v} d^{(j)}(v)
$$

The codegree function $\delta(H, \tau)$ is then defined by

$$
\delta(H, \tau)=2^{\left(\begin{array}{c}
\ell \\
2
\end{array}\right)-1} \sum_{j=2}^{\ell} 2^{-\left(\begin{array}{c}
j-1 \\
2
\end{array}\right)} \delta_{j} .
$$

If $d=0$, define $\delta(H, \tau)=0$.

The precise lemma we will need is a slight variant of Corollary 3.6 from Saxton and Thomason's paper [20]. A similar version was already used in the work of Rödl, Ruciński, and Schacht [19] and we refer the interested reader to that paper for a thorough discussion.

Lemma 3. Let $H$ be an $\ell$-uniform hypergraph on $N$ vertices with average degree $d$. Let $0<\varepsilon<1 / 2$. Suppose that $\tau$ satisfies $\delta(H, \tau) \leqslant \varepsilon / 12 \ell$ ! and $\tau \leqslant 1 / 144 \ell !^{2} \ell$. Then there exists a collection $\mathcal{C}$ of subsets of $V(H)$ such that

( $i)$ for every set $I \subset V(H)$ such that $e(H[I]) \leqslant \varepsilon \tau^{\ell} e(H)$, there is $C \in \mathcal{C}$ with $I \subset C$,

(ii) $e(H[C]) \leqslant \varepsilon e(H)$ for all $C \in \mathcal{C}$,

(iii) $\log |\mathcal{C}| \leqslant 1000 \ell !^{3} \ell \log (1 / \varepsilon) N \tau \log (1 / \tau)$. 
Before we give the proof of Theorem 1, we first describe the $\ell$-uniform hypergraph $H$ to which we will apply Lemma 3.

Construction 4. Given a $k$-uniform hypergraph $F$ with $\ell$ edges, we construct an auxiliary hypergraph $H$ by taking

$$
V(H)=\left(\begin{array}{c}
{[n]} \\
k
\end{array}\right) \quad \text { and } \quad E(H)=\left\{E \in\left(\begin{array}{c}
V(H) \\
\ell
\end{array}\right): E \cong F\right\} .
$$

In other words, the vertices of $H$ are the $k$-tuples of $[n]$ and the edges of $H$ are copies of $F$ in $\left(\begin{array}{c}{[n]} \\ k\end{array}\right)$.

Proof of Theorem 1. Recall that $R=r(F ; q)$, the $q$-color Ramsey number of $F$, and suppose that $F$ has $t$ vertices and $\ell$ edges. Let us fix the following numbers:

$$
\begin{gathered}
\tau=n^{-\frac{1}{2 \ell},} \quad p=1000 R^{k} q \alpha, \quad \alpha=n^{-\frac{1}{2 \ell}+\frac{1}{4 \ell(\ell+1)}} \\
\varepsilon=1 /\left(2 q R^{t}\right), \quad n=\ell^{40 \ell^{2}(\ell+1)}(1000 q)^{8 \ell(\ell+1)} R^{4 k \ell(\ell+1)+4 t \ell}\left(\begin{array}{l}
t \\
k
\end{array}\right)^{4 \ell} .
\end{gathered}
$$

Remark 5. Note that $n$ is bounded above by an expression of the form

$$
2^{c_{1} k \ell^{3} \log (q t \ell)} R^{c_{2} k \ell^{2}+c_{3} t \ell}
$$

as required.

Obviously, $R \geqslant t$ and one can check that $p$ and $n$ satisfy the following conditions, which we will make use of during the course of the proof:

$$
\begin{aligned}
& p \leqslant 1 \\
& n \geqslant\left(24 \cdot 2^{\left(\begin{array}{l}
\ell \\
2
\end{array}\right)} t^{t} q \ell ! R^{t}\right)^{2}, \\
& n>\left(144 \ell !^{2} \ell\right)^{2 \ell}, \\
& n>\ell^{40 \ell^{2}(\ell+1)}, \\
& n>(1000 q)^{8 \ell(\ell+1)} R^{4 k \ell(\ell+1)+4 t \ell}\left(\begin{array}{l}
t \\
k
\end{array}\right)^{4 \ell} .
\end{aligned}
$$

We will show that, with positive probability, a random hypergraph $G \in \mathbb{G}^{(k)}(n, p)$ has the property that every $q$-coloring of its edges contains an induced monochromatic copy of $F$. The proof proceeds in two stages. First, we use Lemma 3 to show that, with probability $1-o(1), G$ has the property that any $q$-coloring of its edges yields many monochromatic copies of $F$. Then we show that some of these monochromatic copies must be induced. 
More formally, let $X$ be the event that there is a $q$-coloring of the edges of $G$ which contains at most

$$
M:=\frac{\varepsilon \tau^{\ell}(n)_{t}}{\operatorname{aut}(F)}
$$

monochromatic copies of $F$ in each color, and let $Y$ be the event that $G$ contains at least $M$ noninduced copies of $F$. Note that if $\bar{X} \cap \bar{Y}$ happens, then, in any $q$-coloring, there are more monochromatic copies of $F$ in one of the $q$ colors than there are noninduced copies of $F$ in $G$. Hence, that color class must contain an induced copy of $F$.

We now proceed to show that the probability $\mathbf{P}(X)$ tends to zero as $n$ tends to infinity. In order to apply Lemma 3, we need to check that $\tau$ and $\varepsilon$ satisfy the requisite assumptions with respect to the $\ell$-uniform hypergraph $H$ defined in Construction 4 . Let $\sigma \subset V(H)$ be arbitrary and define

$$
V_{\sigma}=\bigcup_{v \in \sigma} v \subset[n]
$$

For an arbitrary set $W \subset[n] \backslash V_{\sigma}$ with $|W|=t-\left|V_{\sigma}\right|$, let $\operatorname{emb}_{F}(\sigma, W)$ denote the number of copies $\widetilde{F}$ of $F$ with $V(\widetilde{F})=W \cup V_{\sigma}$ and $\sigma \subset E(\widetilde{F})$. Observe that this number does not actually depend on the choice of $W$, so we will simply use $\operatorname{emb}_{F}(\sigma)$ from now on.

Since there are clearly $\left(\begin{array}{c}n-\left|V_{\sigma}\right| \\ t-\left|V_{\sigma}\right|\end{array}\right)$ choices for the set $W$, we arrive at the following claim.

Claim 1. For any $\sigma \subset V(H)$,

$$
d(\sigma)=\left(\begin{array}{c}
n-\left|V_{\sigma}\right| \\
t-\left|V_{\sigma}\right|
\end{array}\right) \operatorname{emb}_{F}(\sigma)
$$

Let us denote by $t_{j}$ the minimum number of vertices of $F$ which span $j$ edges. From Claim 1, it follows that for any $\sigma \subset V(H)$ with $|\sigma|=j$, we have

$$
d(\sigma)=\left(\begin{array}{c}
n-\left|V_{\sigma}\right| \\
t-\left|V_{\sigma}\right|
\end{array}\right) \operatorname{emb}_{F}(\sigma) \leqslant\left(\begin{array}{c}
n-t_{j} \\
t-t_{j}
\end{array}\right) \operatorname{emb}_{F}(\sigma) .
$$

On the other hand, for a singleton $\sigma_{1} \subset V(H)$, we have $\left|V_{\sigma_{1}}\right|=k$ and therefore $d=d\left(\sigma_{1}\right)$ is such that

$$
\frac{d(\sigma)}{d} \leqslant \frac{\left(\begin{array}{c}
n-t_{j} \\
t-t_{j}
\end{array}\right)}{\left(\begin{array}{c}
n-k \\
t-k
\end{array}\right)} \frac{\operatorname{emb}_{F}(\sigma)}{\operatorname{emb}_{F}\left(\sigma_{1}\right)} \leqslant \frac{\left(\begin{array}{c}
n-t_{j} \\
t-t_{j}
\end{array}\right)}{\left(\begin{array}{c}
n-k \\
t-k
\end{array}\right)}<\left(\frac{n}{t}\right)^{k-t_{j}} .
$$

It then follows from Definition 2 and (2.1) that

$$
\delta_{j}<\frac{(n / t)^{k-t_{j}}}{\tau^{j-1}}<t^{t} n^{k-t_{j}+(j-1) /(2 \ell)} .
$$

Since $t_{j}$ is increasing with respect to $j, t_{2} \geqslant k+1$, and $j \leqslant \ell$, we have $k-t_{j}+\frac{j-1}{2 \ell} \leqslant-1 / 2$. Thus, in view of (2.7), we have

$$
\delta_{j}<t^{t} n^{k-t_{j}+(j-1) /(2 \ell)} \leqslant t^{t} n^{-1 / 2}
$$

for all $2 \leqslant j \leqslant \ell$. 
Using Definition 2 and inequality (2.8), we can now bound the codegree function $\delta(H, \tau)$ by

$$
\delta(H, \tau)=2^{\left(\begin{array}{c}
\ell \\
2
\end{array}\right)-1} \sum_{j=2}^{\ell} 2^{-\left(\begin{array}{c}
j-1 \\
2
\end{array}\right)} \delta_{j} \leqslant 2^{\left(\begin{array}{l}
\ell \\
2
\end{array}\right)-1} t^{t} n^{-1 / 2} \sum_{j=2}^{\ell} 2^{-\left(\begin{array}{c}
j-1 \\
2
\end{array}\right)} \leqslant 2^{\left(\begin{array}{l}
\ell \\
2
\end{array}\right)} t^{t} n^{-1 / 2} .
$$

Since $n$ satisfies (2.3), inequality (2.9) implies that

$$
\delta(H, \tau) \leqslant 2^{\left(\begin{array}{l}
\ell \\
2
\end{array}\right)} t^{t} n^{-1 / 2} \leqslant \frac{\varepsilon}{12 \ell !} .
$$

That is, $\delta(H, \tau)$ satisfies the condition in Lemma 3.

Finally, (2.4) implies that $\tau$ satisfies the condition

$$
\tau=n^{-1 /(2 \ell)}<\frac{1}{144 \ell !^{2} \ell}
$$

Therefore, the assumptions of Lemma 3 are met and we can let $\mathcal{C}$ be the collection of subsets from $V(H)$ obtained from applying Lemma 3. Denote the elements of $\mathcal{C}$ by $C_{1}, C_{2}, \ldots, C_{|\mathcal{C}|}$.

For every choice of $1 \leqslant a_{1}, \ldots, a_{q} \leqslant|\mathcal{C}|$ (not necessarily distinct) let $E_{a_{1}, \ldots, a_{q}}$ be the event that $G \subseteq C_{a_{1}} \cup \cdots \cup C_{a_{q}}$. Next we will show the following claim.

\section{Claim 2.}

$$
\mathbf{P}(X) \leqslant \mathbf{P}\left(\bigvee_{a_{1}, \ldots, a_{q}} E_{a_{1}, \ldots, a_{q}}\right) \leqslant \sum_{a_{1}, \ldots, a_{q}} \mathbf{P}\left(E_{a_{1}, \ldots, a_{q}}\right) .
$$

Proof. Suppose that $G \in X$. By definition, there exists a $q$-coloring of the edges of $G$, say with colors $1,2, \ldots, q$, which contains at most $M$ copies of $F$ in each color. For any color class $j$, let $I_{j}$ denote the set of vertices of $H$ which correspond to edges of color $j$ in $G$. Since each edge in $H\left[I_{j}\right]$ corresponds to a copy of $F$ in color $j$, we have $e\left(H\left[I_{j}\right]\right) \leqslant M$. Note that

$$
M=\varepsilon \tau^{\ell} e(H)
$$

which means that each $I_{j}$ satisfies the condition $((i))$ of Lemma 3. Therefore, for each color class $j$, there must be a set $C_{a_{j}} \in \mathcal{C}$ such that $C_{a_{j}} \supset I_{j}$. Since $G=\bigcup_{j} I_{j}$, this implies that $G \in E_{a_{1}, \ldots, a_{q}}$. Since $G \in X$ was arbitrary, the bound (2.10) follows and the claim is proved.

Owing to Claim 2, we now bound $\mathbf{P}\left(E_{a_{1}, \ldots, a_{q}}\right)$. Recalling the definition of the event $E_{a_{1}, \ldots, a_{q}}$, we note that

$$
\mathbf{P}\left(E_{a_{1}, \ldots, a_{q}}\right)=(1-p)^{\left|V(H) \backslash\left(C_{a_{1}} \cup \cdots \cup C_{a_{q}}\right)\right|} .
$$

Hence, we shall estimate $\left|V(H) \backslash\left(C_{a_{1}} \cup \cdots \cup C_{a_{q}}\right)\right|$ to derive a bound for $\mathbf{P}(X)$ by $(2.10)$. 
Claim 3. For all choices $1 \leqslant a_{1}, \ldots, a_{q} \leqslant|\mathcal{C}|$ we have

$$
\left|V(H) \backslash\left(C_{a_{1}} \cup \cdots \cup C_{a_{q}}\right)\right| \geqslant \frac{1}{2}\left(\frac{n}{R}\right)^{k}
$$

Proof. Let $a_{1}, \ldots, a_{q}$ be fixed and set

$$
\mathcal{A}=\left\{A \in\left(\begin{array}{c}
{[n]} \\
R
\end{array}\right):\left(\begin{array}{c}
A \\
k
\end{array}\right) \subset C_{a_{1}} \cup \cdots \cup C_{a_{q}}\right\} .
$$

By the definition of $R=r(F ; q)$, for each set $A \in \mathcal{A}$ there is an index $j=j(A) \in[q]$ such that $C_{a_{j}}$ contains a copy of $F$ with vertices from $A$. The element $e \in E\left(C_{a_{j}}\right)$ that corresponds to this copy of $F$ satisfies $e \subset\left(\begin{array}{l}A \\ k\end{array}\right)$ and, thus, $\bigcup_{x \in e} x \subset A$. We now give an upper bound for $|\mathcal{A}|$ by counting the number of pairs in

$$
\mathcal{P}=\left\{(e, A) \in \bigcup_{i=1}^{q} E\left(C_{a_{i}}\right) \times \mathcal{A} \text { with } \bigcup_{x \in e} x \subset A\right\} .
$$

On the one hand, we have already established that $|\mathcal{P}| \geqslant|\mathcal{A}|$. On the other hand, for any fixed $e \in E(H)$, we have $\left|\bigcup_{x \in e} x\right|=|V(F)|=t$ and, therefore, there are at most $\left(\begin{array}{c}n-t \\ R-t\end{array}\right)$ sets $A \supset \bigcup_{x \in e} x$. It follows that

$$
\begin{aligned}
&|\mathcal{A}| \leqslant|\mathcal{P}| \leqslant\left|\bigcup_{i=1}^{q} E\left(C_{a_{i}}\right)\right|\left(\begin{array}{l}
n-t \\
R-t
\end{array}\right) \stackrel{((i i))}{\leqslant} q \varepsilon e(H)\left(\begin{array}{c}
n-t \\
R-t
\end{array}\right) \\
& \stackrel{(2.1)}{=} \frac{e(H)}{2 R^{t}}\left(\begin{array}{l}
n-t \\
R-t
\end{array}\right) \leqslant \frac{(n)_{t}}{2 R^{t}}\left(\begin{array}{l}
n-t \\
R-t
\end{array}\right) \leqslant \frac{1}{2}\left(\begin{array}{l}
n \\
R
\end{array}\right) .
\end{aligned}
$$

By definition, each $A \in\left(\begin{array}{c}{[n]} \\ R\end{array}\right) \backslash \mathcal{A}$ satisfies $\left(\begin{array}{c}A \\ k\end{array}\right) \oplus C_{a_{1}} \cup \cdots \cup C_{a_{q}}$. Hence, $V(H) \backslash\left(C_{a_{1}} \cup\right.$ $\left.\cdots \cup C_{a_{q}}\right)$ intersects $\left(\begin{array}{l}A \\ k\end{array}\right)$. Since an element of $V(H)$ can appear in at most $\left(\begin{array}{l}n-k \\ R-k\end{array}\right)$ sets $A$, it follows from (2.13) that there are at least

$$
\frac{1}{2}\left(\begin{array}{l}
n \\
R
\end{array}\right) /\left(\begin{array}{l}
n-k \\
R-k
\end{array}\right) \geqslant \frac{1}{2}\left(\frac{n}{R}\right)^{k}
$$

elements in $V(H) \backslash\left(C_{a_{1}} \cup \cdots \cup C_{a_{q}}\right)$, as required.

In view of Claim 3, our choice of $p=1000 R^{k} q \alpha$, where $\alpha=n^{-1 / 2 \ell+1 / 4 \ell(\ell+1)}$, and (2.11), we have, for any $C_{a_{1}}, \ldots, C_{a_{q}} \in \mathcal{C}$,

$$
\begin{aligned}
\mathbf{P}\left(E_{a_{1}, \ldots, a_{q}}\right) & \leqslant(1-p)^{(n / R)^{k} / 2} \\
& \leqslant \exp \left(-p n^{k} / 2 R^{k}\right)=\exp \left(-\left(1000 R^{k} q \alpha\right) n^{k} / 2 R^{k}\right) \\
& =e^{-500 q \alpha n^{k}} \leqslant e^{-1000 q \alpha N},
\end{aligned}
$$


where, in the last step, we used $N=\left(\begin{array}{l}n \\ k\end{array}\right) \leqslant \frac{n^{k}}{2}$. Therefore, (2.10) and (2.14) together with the bound on $|\mathcal{C}|$ given by Lemma $3(($ iii $))$ imply that

$$
\begin{aligned}
\mathbf{P}(X) & \leqslant \sum_{C_{a_{1}}, \ldots, C_{a_{q}} \in \mathcal{C}} \mathbf{P}\left(E_{a_{1}, \ldots, a_{q}}\right) \leqslant|\mathcal{C}|^{q} e^{-1000 q \alpha N} \\
& \leqslant \exp \left(1000 q \ell !^{3} \ell \log (1 / \varepsilon) N \tau \log (1 / \tau)-1000 q \alpha N\right) \\
& =\exp \left(1000 q N \tau\left(\ell !^{3} \ell \log (1 / \varepsilon) \log (1 / \tau)-\alpha / \tau\right)\right) \\
& \leqslant \exp \left(1000 q N \tau\left(\ell !^{3} \log ^{2} n-n^{1 /(4 \ell(\ell+1))}\right)\right) \leqslant 1 / 4
\end{aligned}
$$

where we used that $n$ satisfies (2.5).

Now, by Markov's inequality, with probability at least $1 / 2$, the number of noninduced copies of $F$ in $G$ will be at most twice the expected number of copies, which is fewer than

$$
\begin{aligned}
2 p^{\ell+1} \frac{(n)_{t}}{\operatorname{aut}(F)}\left(\begin{array}{l}
t \\
k
\end{array}\right) & =2(1000 q)^{\ell+1} R^{k(\ell+1)} n^{-1 / 2-1 /(4 \ell)} \frac{(n)_{t}}{\operatorname{aut}(F)}\left(\begin{array}{l}
t \\
k
\end{array}\right) \\
& <\frac{1}{2 q R^{t}}\left(n^{-1 /(2 \ell)}\right)^{\ell} \frac{(n)_{t}}{\operatorname{aut}(F)}=\varepsilon \tau^{\ell} \frac{(n)_{t}}{\operatorname{aut}(F)}=M
\end{aligned}
$$

where the inequality above follows from (2.6). In other words, $\mathbf{P}(\bar{Y}) \geqslant 1 / 2$ and, therefore, $\mathbf{P}(\bar{X} \cap \bar{Y}) \geqslant 1 / 4$, so there exists a graph $G$ such that $\bar{X} \cap \bar{Y}$ holds. By our earlier observations, this completes the proof.

\section{§3. CONCLUding REMARKS}

Beginning with Fox and Sudakov [10], much of the recent work on induced Ramsey numbers for graphs has used pseudorandom rather than random graphs for the target graph $G$. The results of this paper rely very firmly on using random hypergraphs. It would be interesting to know whether comparable bounds could be proved using pseudorandom hypergraphs.

It would also be interesting to prove comparable bounds for the following variant of the induced Ramsey theorem, first proved by Nešetřil and Rödl [15]: for every graph $F$, there exists a graph $G$ such that every $q$-coloring of the triangles of $G$ contains an induced copy of $F$ all of whose triangles receive the same color. By taking $F=K_{t}$ and $q=4$, we see that $|G|$ may need to be double exponential in $|F|$. We believe that a matching double-exponential upper bound should also hold.

\section{REFERENCES}

[1] F. G. Abramson and L. A. Harrington, Models without indiscernibles, J. Symbolic Logic 43 (1978), no. 3, 572-600, DOI 10.2307/2273534. MR503795 ^1

[2] J. Balogh, R. Morris, and W. Samotij, Independent sets in hypergraphs, J. Amer. Math. Soc. 28 (2015), no. 3, 669-709, DOI 10.1090/S0894-0347-2014-00816-X. MR3327533 ^1 
[3] D. Conlon, J. Fox, and B. Sudakov, On two problems in graph Ramsey theory, Combinatorica 32 (2012), no. 5, 513-535, DOI 10.1007/s00493-012-2710-3. MR3004807 $\uparrow 1$

[4] _ An improved bound for the stepping-up lemma, Discrete Appl. Math. 161 (2013), no. 9, 1191-1196, DOI 10.1016/j.dam.2010.10.013. MR3030610 ^1

[5] _ Recent developments in graph Ramsey theory, Surveys in combinatorics 2015, London Math. Soc. Lecture Note Ser., vol. 424, Cambridge Univ. Press, Cambridge, 2015, pp. 49-118, DOI 10.1017/CBO9781316106853.003. $\uparrow 1$

[6] W. Deuber, Generalizations of Ramsey's theorem, Infinite and finite sets (Colloq., Keszthely, 1973; dedicated to P. Erdős on his 60th birthday), Vol. I, North-Holland, Amsterdam, 1975, pp. 323-332. Colloq. Math. Soc. János Bolyai, Vol. 10. MR0369127 $\uparrow 1$

[7] P. Erdős, On some problems in graph theory, combinatorial analysis and combinatorial number theory, Graph theory and combinatorics (Cambridge, 1983), Academic Press, London, 1984, pp. 1-17. MR777160 11

[8] P. Erdős, A. Hajnal, and L. Pósa, Strong embeddings of graphs into colored graphs, Infinite and finite sets (Colloq., Keszthely, 1973; dedicated to P. Erdős on his 60th birthday), Vol. I, North-Holland, Amsterdam, 1975, pp. 585-595. Colloq. Math. Soc. János Bolyai, Vol. 10. MR0382049 11

[9] P. Erdös and R. Rado, Combinatorial theorems on classifications of subsets of a given set, Proc. London Math. Soc. (3) 2 (1952), 417-439. MR0065615 ^1

[10] J. Fox and B. Sudakov, Induced Ramsey-type theorems, Adv. Math. 219 (2008), no. 6, 1771-1800, DOI 10.1016/j.aim.2008.07.009. MR2455625 ^3

[11] _ Density theorems for bipartite graphs and related Ramsey-type results, Combinatorica 29 (2009), no. 2, 153-196. MR2520279 ^1

[12] R. L. Graham, B. L. Rothschild, and J. H. Spencer, Ramsey theory, 2nd ed., Wiley-Interscience Series in Discrete Mathematics and Optimization, John Wiley \& Sons, Inc., New York, 1990. A Wiley-Interscience Publication. MR1044995 11

[13] Y. Kohayakawa, H. J. Prömel, and V. Rödl, Induced Ramsey numbers, Combinatorica 18 (1998), no. 3, 373-404, DOI 10.1007/PL00009828. MR1721950 ^1

[14] R. Nenadov and A. Steger, A short proof of the random Ramsey theorem, Combin. Probab. Comput. 25 (2016), no. 1, 130-144, DOI 10.1017/S0963548314000832. MR3438289 ^1

[15] J. Nešetřil and V. Rödl, Partitions of subgraphs, Recent advances in graph theory (Proc. Second Czechoslovak Sympos., Prague, 1974), Academia, Prague, 1975, pp. 413-423. MR0429655 33

[16] _ Partitions of finite relational and set systems, J. Combinatorial Theory Ser. A 22 (1977), no. 3, 289-312. MR0437351 11

[17] F. P. Ramsey, On a problem of formal logic, Proc. London Math. Soc. (2) 30 (1930), no. 1, 264-286, DOI $10.1112 / \mathrm{plms} / \mathrm{s} 2-30.1 .264$. $\uparrow 1$

[18] V. Rödl, The dimension of a graph and generalized Ramsey theorems, Master's thesis, Charles University, 1973. $\uparrow 1$

[19] V. Rödl, A. Ruciński, and M. Schacht, An exponential-type upper bound for Folkman numbers, Combinatorica, DOI 10.1007/s00493-015-3298-1. to appear. $\uparrow 1,2$

[20] D. Saxton and A. Thomason, Hypergraph containers, Invent. Math. 201 (2015), no. 3, 925-992, DOI 10.1007/s00222-014-0562-8. MR3385638 ^1, 2 
Mathematical Institute, University of Oxford, Oxford, United Kingdom

E-mail address: david.conlon@maths.ox.ac.uk

Department of Mathematics and Computer Science, Emory University, Atlanta, USA

E-mail address: \{ddellam, slafleu, rodl\}@emory.edu

Fachbereich Mathematik, Universität Hamburg, Hamburg, Germany

E-mail address: schacht@math.uni-hamburg.de 